\title{
Is there a simple and less invasive way to accurately diagnose acute pyelonephritis?
}

\section{Seong Heon Kim, MD \\ Department of Pediatrics, Pusan National University Children's Hospital, Yangsan, Korea}

Urinary tract infections (UTIs) occurring at a young age of 24 months or less are among the most common bacterial infections, and urine culture tests have been recognized as the golden standard of diagnosis; however, the diagnosis is often uncertain. ${ }^{1,2)}$ Although most guidelines state that urine tests must be performed before the start of antibiotic treatment, it is not uncommon to observe otherwise in a clinical situation. In this case, urine culture tests usually show negative results, even in cases of definite UTI. Using a urine bag used to obtain urine sample from a child who is not toilet-trained often leads to false-positive results on urine culture tests. Fig. 1 shows the limitations and pitfalls of diagnosing acute pyelonephritis (APN) using urine culture. ${ }^{1,3)}$

A dimercaptosuccinic acid (DMSA) scan is a reliable test that can detect both APN and late renal parenchymal scarring. However, the test is expensive and involves radiation exposure and sedation. Moreover, DMSA scan cannot replace voiding cystourethrography in the diagnosis of vesicoureteral reflux. The proper diagnostic methods and imaging tests for a child with UTI remain controversial.

In the recent issue of the Korean Journal of Pediatrics, Lee and Rhie ${ }^{4)}$ published an article titled "Urine culture as a tool for the diagnosis of acute pyelonephritis in children had better be reconsidered: New challenging method of diagnosing acute pyelonephritis." This article describes several limitations of the traditional golden standard, urine culture, for diagnosing APN and the invasiveness of collecting techniques such as suprapubic aspiration and catheterization. Lee et al. introduces the new approaches to diagnosing APN using a combination of urinalysis, white blood cell (WBC) count, several inflammatory markers, other fever focus, past history of UTI, sex, urine protein, and urine $\mathrm{Na} / \mathrm{K}$ ratio, among others. Lee and Rhie $^{4)}$ describe 3 major causes of febrile UTI (APN, pyelitis, lower UTI with other fever focus),

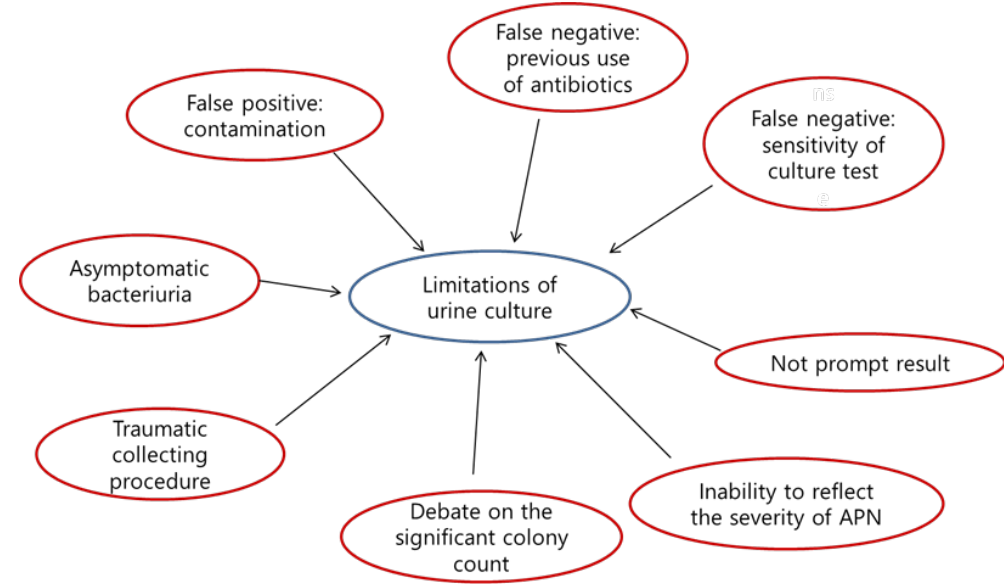

Fig. 1. Limitations and pitfalls in the diagnosis of acute pyelonephritis (APN) made using urine culture.
Corresponding author: Seong Heon Kim, MD Department of Pediatrics, Pusan National University Children's Hospital, 20 Geumo-ro, Mulgeumeup, Yangsan 50612, Korea

Tel: +82-55-360-3163

Fax: +82-55-360-2181

E-mail: pedksh@gmail.com

https://orcid.org/0000-0001-8003-3010

Received: 1 October, 2019

Revised: 14 November, 2019

Accepted: 15 November, 2019

See the article "Reconsideration of urine culture for the diagnosis of acute pyelonephritis in children: a new challenging method for diagnosing acute pyelonephritis" in Volume 62 on page 433. 
of which only APN can be associated with renal damage, and states that urine culture tests do not differentiate among them. This article focuses more on APN despite controversy regarding the diagnosis of febrile UTIs such as leukocyturia, colony count, and asymptomatic bacteriuria. $^{1,3,5)}$

A DMSA scan or computed tomography is the most accurate method of diagnosing APN, but due to radiation problems, it must be used with caution. In addition to the method by Lee and Rhie, recent studies on serum markers to diagnose APN have shown promising results. ${ }^{6-8)}$ Among them, plasma neutrophil gelatinaseassociated lipocalin (NGAL) seems the most noteworthy, although additional studies are needed. Kim et al. ${ }^{7)}$ reported that plasma NGAL could be more useful than serum procalcitonin, C-reactive protein, and WBC levels for identifying APN in children. In their study, a multivariate analysis revealed that only plasma NGAL level was an independent predictor of APN. At the best cutoff values of all examined biomarkers for identifying APN, sensitivity (86\%), specificity (85\%), positive predictive value (81\%), and negative predictive value (89\%) of plasma NGAL levels were the highest. ${ }^{7)}$

This article by Lee and Rhie $^{4)}$ has great significance since it presents a method for diagnosing APN using simple tests and history rather than an invasive urine culture test that requires a few days for confirmation. Although this paper presents the author's new approach to diagnosing APN associated with renal damage, it is based not on sufficient evidence but rather mainly on expert opinion and is not currently widely used. Thus, further studies using this method are necessary to confirm its clinical usefulness to discriminate between APN and other conditions.

In conclusion, this article by Lee and Rhie ${ }^{4)}$ presents a good alternative of simple and less invasive tests for the diagnosis of APN. However, clinicians should be aware of several controversies related to the diagnosis and imaging of APN to make the right decisions. Furthermore, efforts should be continued to diagnose APN using easy tests while avoiding overtreatment.

\section{Conflicts of interest}

No potential conflict of interest relevant to this article was reported.

\section{References}

1. Okarska-Napierala M, Wasilewska A, Kuchar E. Urinary tract infection in children: Diagnosis, treatment, imaging - comparison of current guidelines. J Pediatric Urol 2017;13:567-73.

2. Subcommittee on Urinary Tract Infection, Steering Committee on Quality Improvement and Management, Roberts KB. Urinary tract infection: clinical practice guideline for the diagnosis and management of the initial UTI in febrile infants and children 2 to 24 months. Pediatrics 2011;128:595-610.

3. Roberts KB, Wald ER. The diagnosis of UTI: colony count criteria revisited. Pediatrics 2018;141(2). pii: e20173239. https://doi.org/ 10.1542/peds.2017-3239.

4. Lee JH, Rhie SK. Reconsideration of urine culture for the diagnosis of acute pyelonephritis in children: a new challenging method for diagnosing acute pyelonephritis. Korean J Pediatr 2019;62:433-37.

5. Tullus K. New Italian guidelines on UTI are one step forward. Acta Paediatr 2019 Sep 28 [Epub]. https://doi.org/10.1111/apa.15037.

6. Shaikh N, Borrell JL, Evron J, Leeflang MM. Procalcitonin, C-reactive protein, and erythrocyte sedimentation rate for the diagnosis of acute pyelonephritis in children. Cochrane Database Syst Rev 2015;1: CD009185.

7. Kim BK, Yim HE, Yoo KH. Plasma neutrophil gelatinase-associated lipocalin: a marker of acute pyelonephritis in children. Pediatr Nephrol 2017;32:477-84.

8. Yun BA, Yang EM, Kim CJ. Plasma neutrophil gelatinase-associated lipocalin as a predictor of renal parenchymal involvement in infants with febrile urinary tract infection: a preliminary study. Ann Lab Med 2018;38:425-30. 